Service social

\title{
Le partenaire invisible : les conditions de travail dans l'évaluation et l'intervention sociales
}

\section{Maurice Moreau et Michelle Desrosiers}

Volume 35, numéro 3, 1986

Les jeunes et le travail social

URI : https://id.erudit.org/iderudit/706320ar

DOI : https://doi.org/10.7202/706320ar

Aller au sommaire du numéro

Éditeur(s)

École de service social de l'Université Laval

ISSN

1708-1734 (numérique)

Découvrir la revue

Citer ce document

Moreau, M. \& Desrosiers, M. (1986). Le partenaire invisible : les conditions de travail dans l'évaluation et l'intervention sociales. Service social, 35(3), 375-385. https://doi.org/10.7202/706320ar
Résumé de l'article

Cet article soulève l'importance des caractéristiques personnelles et socio-démographiques sur la position de l'individu dans l'organisation et la division du travail, et du rôle que jouent les conditions de travail dans les problèmes sociaux.

Les auteurs présentent, en seconde partie, quelques pistes d'intervention en milieu de travail, attirant l'attention sur le rôle que peut y jouer l'intervenant social. 


\section{COMMENTAIRES \\ ET DOCUMENTS}

MOREAU, Maurice, professeur à l'École de service social de l'Université de Montréal.

DESROSIERS, Michelle, travailleuse sociale au Carrefour Ubald-Villeneuve à SainteFoy.

\section{Le partenaire invisible : les conditions de travail dans l'évaluation et l'intervention sociales}

\section{Maurice Moreau Michelle Desrosiers}

Plusieurs approches contemporaines en travail social laissent entendre que seuls les dynamiques individuelles et/ou familiales et les facteurs d'ordre psychologique sont à l'origine des problèmes, même matériels, des personnes qui consultent les services sociaux (Moreau, 1982). En effet, la plupart de ces approches ne tentent pas d'explorer les liens qui peuvent exister entre les façons d'être d'un individu et la place objective qu'il occupe dans l'organisation et la division sociale et sexuelle du travail. De ce fait, ce sont uniquement les individus, et non leurs conditions d'emploi et de vie, qui risquent d'être remis en question dans l'évaluation et l'intervention sociales. Nous soutenons que la place qu'occupe une personne dans l'organisation et la division du travail affecte les conditions de vie qui en découlent. Dans notre société, cette place varie selon la classe sociale, l'âge, le sexe, l'orientation sexuelle, la race, l'ethnie, la nationalité, la santé physique et mentale et le statut civil. Ces caractéristiques personnelles et socio-démographiques peuvent 
avoir un effet sur le niveau objectif d'infériorisation sociale que subit un individu. Enfin, nous postulons qu'il est primordial de tenir compte des caractéristiques des conditions de travail et de vie de chaque personne et du degré d'infériorisation sociale qui en découle pour comprendre l'ensemble des facteurs qui interviennent sur la santé physique, les façons de penser, d'agir et de se sentir envers soi-même et les autres.

Dans cet article, nous élaborons cette hypothèse et soulignons les implications de cette perspective pour l'évaluation sociale.

\section{Quand on est exclu}

\section{Les chômeurs, les assistés sociaux et les handicapés}

Il existe, dans notre société, deux sortes de travail : salarié, c'est-àdire productif, et non salarié, c'est-à-dire de reproduction sociale, considéré comme non directement productif (Corrigan et Leonard, 1978). La valeur sociale des personnes est ainsi généralement reliée à leur capacité de produire ou de reproduire, et des restrictions sociales importantes sont imposées sur l'attribution du travail et les conditions qui s'y rattachent.

C'est ainsi, par exemple, que plusieurs chômeurs et assistés sociaux, étant exclus du marché du travail défini comme productif, se sentent et sont considérés inférieurs. C'est également ainsi que plusieurs personnes handicapées physiques ou mentales se perçoivent inadéquates; elles sont le plus souvent exclues de la production ou, si elles sont inclues, travaillent en usine protégée, exploitées au plan financier. Qu'arrivet-il à la perception et à l'estime de soi, aux comportements que l'on développe quand on est chômeur, assisté social ou handicapé et donc structurellement exclu d'une source de valorisation sociale majeure? Quels sont les liens entre le fait d'avoir moins d'argent, d'être moins mobile socialement, d'avoir à vivre dans l'imprévisibilité et le sentiment d'être constamment inquiet ? L'insuffisance de revenu ne peut qu'aggraver les problèmes de santé. Quand on est assisté social ou chômeur, on a plus de temps libre mais moins d'argent pour se divertir et se payer des vacances. On peut donc être contraint à passer plus de temps dans des espaces (quartier, logement) inadéquats. On risque aussi de vivre socialement plus isolé. Est-ce que le fait d'être supervisé, scruté et régulièrement sous la loupe des agents d'aide sociale ou de l'assurancechômage affecte les comportements ? Comment se sent-on quand on est handicapé et donc objet de pitié ? Comment se comporte-t-on 
quand on est méprisé ou rejeté parce qu'on est chômeur ou assisté social ? Autant de questions à fouiller si l'on veut tenir compte, dans nos évaluations sociales, des liens entre le vécu personnel et la place objective occupée dans la division et l'organisation du travail.

\section{Les jeunes et les personnes âgées}

Au lieu de concevoir la vie comme un continuum, notre société sépare arbitrairement les personnes les unes des autres, selon leur âge : de la naissance à 18 ans ou 21 ans, on les considère "trop jeunes pour "; de 21 à 65 ans, période jugée productive, on les dit "assez vieilles pour"; enfin, de 65 ans à leur mort, elles sont perçues "trop vieilles pour ". Les jeunes sont socialement mal vus parce qu'ils coûtent trop cher et contestent tout ; les vieux, parce qu'ils ne rapportent plus.

C'est ainsi qu'ayant à rivaliser pour un meilleur statut social, des emplois et des ressources qui se font de plus en plus rares, beaucoup de jeunes et de personnes âgées en viennent à se méfier les uns des autres, en dépit du fait qu'ils ont énormément en commun. Les deux groupes vivent un certain degré de conflit avec la génération d'âge moyen; ils ont tous deux des problèmes de revenu et d'emploi à cause de la discrimination selon l'âge ; aucun des deux n'est pris au sérieux. On dit aux aînés: "on ne fait plus les choses de cette façon ", et aux jeunes: "vous ne savez pas de quoi vous parlez". Les deux groupes sont privés d'activités professionnelles et sociales durables et significatives; ils subissent des changements physiques importants et enfin, quoiqu'il s'agisse de drogues différentes provenant de sources différentes, les deux groupes sont aussi aux prises avec de sérieux problèmes sur ce plan. Écartés socialement les uns des autres à cause de leur âge, et contraints à la passivité, est-il étonnant qu'autant de jeunes et de personnes âgées souffrent d'ennui, de dépression et que certains en viennent jusqu'au suicide?

Il est important de souligner que cette cloison entre les générations n'a pas toujours existé, qu'elle a été socialement et artificiellement créée, et qu'elle a varié, historiquement, selon les besoins de garder, comme réserve de main-d'œuvre, une certaine partie de la population. Nous vivons donc dans une société qui opprime et infériorise les personnes handicapées, les chômeurs, les assistés sociaux, les jeunes et les personnes âgées, parce qu'on associe leur valeur sociale à leurs capacités de produire. Comment ne pas tenir compte de ces éléments dans nos évaluations sociales? 


\section{Les femmes et les hommes}

Une réflexion sur l'organisation du travail rend vite compte d'autres divisions importantes: celles qui sont basées sur le sexe, l'orientation sexuelle et le statut civil. En effet, ce sont encore surtout les hommes qui œuvrent dans la production alors que les femmes assument la reproduction sociale, qu'elles aient ou non un emploi rémunéré hors du foyer (Armstrong, 1978; Meisner, 1975). Cette division arbitraire contribue à surcharger les femmes qui se retrouvent, si elles travaillent à l'extérieur, avec un double fardeau. Existerait-il un lien entre le sentiment de futilité, d'aberration et de dépression que plusieurs femmes vivent et le fait d'avoir à vaquer seules aux travaux domestiques, activités isolées, non rémunérées, répétitives, monotones et interminables (Oakley, 1974) ? Y aurait-il un lien entre le fait que plusieurs d'entre elles se sentent vidées affectivement, et celui d'avoir à porter, à elles seules, le poids de la responsabilité de la vie affective des hommes et des enfants? La peur de ne rien réussir seules ou celle d'être blâmées pour avoir réussi, la crainte de déplaire et l'insécurité chronique de plusieurs femmes seraient-elles ultimement reliées à la dépendance économique à laquelle la plupart sont contraintes? La division sexuelle du travail favorise la création de liens parfois malsains entre mères et enfants surtout quand, faute d'options, elles sur-investissent en eux ; ces derniers représentent les seuls fruits de leur travail (Chodorow, 1978; Gail, 1968 ; Nunes et White, 1972). Elle contribue de plus, à notre avis, à rendre difficile le développement de liens affectifs significatifs entre les hommes et leurs enfants. Cette distance sociale est un facteur qui ne peut qu'objectiver les rapports entre eux et ainsi favoriser le développement de liens incestueux entre pères et enfants (Chodorow, 1978).

La division sexuelle du travail est maintenue; elle rend plus difficile le travail salarié pour les femmes tout en privilégiant une forme familiale précise: celle où l'homme a un emploi rémunéré à l'extérieur et répond aux besoins économiques de sa femme et de ses enfants, alors que celle-ci travaille sans salaire à la maison, où elle conserve la responsabilité des tâches domestiques, des enfants et de la réussite de la vie familiale. Parmi les mécanismes sociaux qui maintiennent cette forme familiale, on identifie, entre autres, le manque de garderies, de foyers pour femmes battues et d'emplois pour les femmes. Nous continuons donc à observer des discriminations pour tous les types de famille autres que la famille hétérosexuelle, isolée, nucléaire, traditionnelle. La typologie de la maternité, développée par Helen Levine et Alma Estable (1981), est éclairante à ce sujet. Comme le tableau suivant l'indique, on y retrouve neuf catégories de mères: les mères qui sont bonnes, normales et adéquates; celles qui sont considérées dangereuses; 
celles qui sont définies comme mauvaises, déviantes ou inadéquates. Selon Levine et Estable, plus la maternité se situe hors des normes reconnues d'hétérosexualité et hors des rôles familiaux traditionnels, plus elle est considérée mauvaise. Comment pouvons-nous continuer, comme travailleurs sociaux, à dénoncer la négligence des enfants sans également remettre en question cette catégorisation?

\section{TABLEAU 1}

\section{Typologie de la maternité.}

\begin{tabular}{|c|c|}
\hline $\begin{array}{l}\text { Bonnes } \\
\text { Normales } \\
\text { Adéquates }\end{array}$ & $\begin{array}{l}\text { 1. Mariée, } 2 \text { enfants, le mari travaille, la mère est à la } \\
\text { maison. } \\
\text { 2. Veuve (catégorie encore respectable - a droit à une } \\
\text { pension... sans questions). }\end{array}$ \\
\hline $\begin{array}{l}\text { Zone grise } \\
\text { et } \\
\text { dangereuse }\end{array}$ & $\begin{array}{l}\text { 3. Mère célibataire, séparée, divorcée ou abandonnée par } \\
\text { son conjoint (doit prouver son besoin économique, est } \\
\text { scrutée et sujette à la dégradation). }\end{array}$ \\
\hline \multirow{6}{*}{$\begin{array}{l}\text { Mauvaises } \\
\text { Anormales } \\
\text { Inadéquates }\end{array}$} & 4. Mère célibataire qui garde son enfant. \\
\hline & 5. Mère célibataire qui donne son enfant en adoption. \\
\hline & $\begin{array}{l}\text { 6. Mère célibataire qui décide d'avoir un enfant sans l'im- } \\
\text { plication d'un conjoint légal ou autre. }\end{array}$ \\
\hline & 7. Mère qui perd la garde de ses enfants. \\
\hline & $\begin{array}{l}\text { 8. Mère qui confie volontairement la garde de ses enfants } \\
\text { au père ou à l'État. }\end{array}$ \\
\hline & $\begin{array}{l}\text { 9. Mère lesbienne (toujours susceptible de se faire enlever } \\
\text { ses enfants). }\end{array}$ \\
\hline
\end{tabular}

Afin d'assurer le maintien de cette forme familiale et de la division sexuelle du travail qui la supporte, il importe que les enfants soient socialisés différemment selon leur sexe. Ainsi les garçons apprennent à rivaliser, à être servis, à être rationnels, à agir, à dominer et à apparaître forts en tout temps, ce qui les amène à cacher leurs faiblesses. En revanche, les filles apprennent à coopérer, à servir, à être émotives, à réagir, à se soumettre et à apparaître faibles en tout temps, ce qui les amène à cacher leurs forces. Comme les travaux de Broverman (1970) l'ont démontré, les comportements que la plupart des thérapeutes considèrent désirables chez les garçons sont des comportements adultes et normaux alors que les comportements qu'ils considèrent désirables 
chez les filles sont ceux associés aux enfants et donc à l'immaturité et au sous-développement psychologique. De plus, cette socialisation amène les hommes et les femmes à nier et à détester une partie importante d'eux-mêmes tout en recherchant cette partie, niée et détestée, chez le sexe opposé. Cette dynamique ne peut qu'entraîner des problèmes importants de relations entre les hommes et les femmes.

\section{Les classes sociales}

Dans notre système social, politique et économique, il importe que certaines personnes pensent et donnent des directives, et que cette fonction soit plus rémunérée et plus reconnue socialement, alors que d'autres doivent agir, suivre ces ordres et être moins payés et valorisés pour leurs efforts. Nous référons ici à la division du travail selon les classes sociales. Si la classe dirigeante travaille intellectuellement et commande, la classe ouvrière, elle, travaille manuellement et exécute. Existerait-il un lien entre le fait de subir des ordres sans pouvoir maugréer, et le fait de compenser au foyer en écrasant les autres? Existerait-il un lien entre le fait de donner des ordres à la journée longue, et vouloir se retirer de cette responsabilité au foyer? Enfin, quelles sont, à long terme, pour le travailleur, les conséquences psychologiques de la déqualification et de la dégradation croissantes du travail dont parle Braverman (1974)?

L'école joue un rôle très important dans la reproduction contemporaine des classes sociales et il vaut la peine de s'y attarder. Un de ses objectifs principaux est, en effet, de reproduire la force de travail hiérarchisée requise de notre système social, politique et économique. Les écoles sont modelées sur deux sortes de bureaucraties : la bureaucratie de l'usine, pour les enfants de classe ouvrière, et la bureaucratie de la corporation, pour les enfants de classe privilégiée (Leonard, 1984; Ryan, 1982). Si les enfants de la classe privilégiée jouissent de plus de contrôle sur leurs projets, leurs horaires et les ressources de leurs écoles que les enfants de la classe ouvrière, les amenant donc à l'habitude du contrôle, il demeure cependant que tous les enfants des écoles contemporaines, indépendamment de leur classe sociale, sont énormément sur-contrôlés par des règlements et des précautions sans bornes. On y contrôle le savoir - enseignant que ce sont avant tout les qualités individuelles qui différencient les personnes et qui justifient leur supériorité. On y contrôle le temps: l'heure de manger, de se reposer et même d'uriner - inculquant avant tout l'importance capitale de la ponctualité et de la subordination à l'autorité. On y contrôle les mouvements : l'endroit où l'on doit s'asseoir, avec qui on peut parler, 
marcher et se tenir. Si l'on découvre des activités de groupe qui ne sont pas sous l'égide d'une autorité - mais sous le leadership d'un enfant on soupçonne trop souvent qu'il s'agit là d'une source de contestation d'une règle quelconque et on intervient. On y contrôle en particulier la place que doit occuper chacun par l'assignation de notes. C'est ainsi que tous sont passés au crible et subjectivement étiquetés. Comme de la viande, les étudiants sont classés "de catégorie surchoix, choix, bon ou utilité ". L'importance de ce processus est telle que si les notes d'un professeur sont trop élevées, ce dernier risque d'être jugé ou trop permissif, ou subversif, ou mauvais enseignant. Dans la réussite, on insiste sur l'importance de la différence et de la compétition entre étudiants et étudiantes. Dans ce climat, la coopération avec ses amis est presque défendue et, quand cela se produit, on la qualifie de trichage. Est-il surprenant que ce contexte finisse par rendre plusieurs jeunes déprimés, agressifs ou encore repliés sur eux-mêmes ? Faut-il les blâmer, dans de telles conditions, d'avoir le goût de décrocher de l'école et de la société?

\section{Le racisme}

Enfin, il importe de souligner, dans cette réflexion sur l'organisation du travail, que ce sont plus particulièrement les personnes de race noire, rouge et jaune, collectivement considérées inférieures, qui sont les plus susceptibles de se retrouver parmi celles qui exécutent les tâches subalternes, dévalorisées et les moins payées. L'organisation et la division du travail sont donc aussi empreintes de racisme.

Lorsqu'on insiste sur l'impact des forces structurelles extérieures à l'individu, dans la création de leurs situations problèmes, on oublie que les façons plus ou moins conscientes de se sentir, de penser, d'agir et d'être des personnes contribuent au maintien, à la légitimation et à la reproduction de ces structures (Adam, 1978; Ryan, 1982; Shatz, 1973 ; Steiner, 1974). Toutefois, nous croyons que le fait de tenir compte, dans un premier temps, de l'impact structurel des conditions de travail et de vie dans la situation problème d'un individu et de tenter d'intervenir d'abord à ce niveau, dans la mesure du possible (Galper, 1980 ; Leonard, 1975 ; Moreau, 1979), empêche le travailleur social de contribuer à en faire un bouc émissaire dans une situation qui ne relève pas exclusivement de lui.

Mais, en plus d'être attentifs au rôle des conditions de travail dans leurs évaluations sociales, les intervenants sociaux peuvent-ils entreprendre d'autres actions concrètes? À notre avis, deux pistes d'intervention semblent possibles. 


\section{Pistes d'intervention}

\section{L'intervention syndicale}

S'il existe un regroupement qui s'est historiquement préoccupé du bien-être des travailleurs, et non seulement de leur rendement et des profits qu'ils pourraient rapporter à l'entreprise, c'est bien le mouvement syndical. C'est pourquoi l'un des actes concrets que peut poser un intervenant social est de considérer et d'avoir recours aux syndicats comme ressources pour les travailleurs qui vivent des conditions de travail difficiles. Il peut, lui aussi, se syndiquer et supporter généralement le mouvement syndical. Toutefois, plusieurs syndicats en Amérique du Nord sont accusés de ne pas respecter le processus démocratique dans leurs méthodes de fonctionnement (Srinivas, 1980). L'intervenant social devra donc demeurer critique et dénoncer tout abus de pouvoir, à l'intérieur des syndicats, vis-à-vis des membres. Il s'opposera ainsi à toute méthode de fonctionnement ou programmes syndicaux sexistes, racistes ou antidémocratiques qui reproduiraient une forme d'oppression ressemblant finalement à celle dont les travailleurs sont victimes de la part des patrons.

L'intervenant social peut également exercer un rôle important comme personne-ressource pour le syndicat (Desmarais, 1982). II peut agir comme agent de sensibilisation et de conscientisation, pour le mouvement syndical ou au sein de ce dernier. II peut également fournir au syndicat des outils d'animation et d'évaluation afin d'aider les syndiqués à explorer les liens entre leurs problèmes psychologiques et interpersonnels et leurs conditions de travail.

\section{L'intervention patronale}

Plusieurs facteurs ont récemment forcé l'entreprise à s'intéresser de plus près à la qualité de vie et au bien-être des travailleurs; parmi ceux-ci, on note des relations industrielles de plus en plus perturbées, ainsi qu'une baisse accrue de la productivité et des profits causée en partie par un taux très élevé de retards au travail, de mobilité du personnel et d'absentéisme relié, lui, très souvent, à une baisse de moral chez les travailleurs, à l'alcoolisme et à la consommation d'autres drogues. Les divisions canadiennes de plusieurs grandes multinationales, comme les compagnies General Electric, Shell Oil et Union Carbide, et de nombreuses compagnies canadiennes telles Inco Metals, MacMillan Bloëdel, Miracle Food Mart, C.I.L., Canadair et Steinberg, pour n'en 
nommer que quelques-unes, ont en effet récemment mis sur pied de nouveaux projets visant à humaniser les milieux de travail (Srinivas, 1980). Parmi les plus conventionnels, on trouve les programmes d'aide aux employés. Parmi les plus avant-gardistes, il y a les comités de gestion paritaire travailleurs-patrons et les groupes de travail semi-autonomes. Plusieurs de ces projets accordent un plus grand contrôle aux travailleurs : sur la gestion de leur temps, permettant, par exemple, la compression et la flexibilité des horaires de travail; sur la nature des emplois qu'ils effectuent, acceptant, entre autres, la rotation et le partage, l'élargissement et l'enrichissement de certain emplois ainsi que la conception d'emplois favorisant le développement maximal de toutes les capacités du travailleur.

Si l'intervenant social se doit d'être ouvert et positif face à de tels projets de collaboration entre le patronat et les travailleurs, en autant qu'ils améliorent les conditions de travail de ces derniers, il doit rester vigilant à leur égard. Car, en pratique, plusieurs projets de collaboration de cette nature sont vendus à des compagnies par des consultants en gestion dans le but d'empêcher la syndicalisation des employés. De plus, là où de tels programmes sont en place et où les employés sont déjà syndiqués, ils risquent de miner les revendications les plus fondamentales et conflictuelles à la base des intérêts syndicaux : le partage équitable des gains économiques qui viennent d'une productivité accrue de la part des travailleurs, et le partage du droit de propriété. Enfin, la cogestion d'une entreprise peut exiger des travailleurs cogestionnaires qu'ils entérinent des transferts, des mises à pied et des rétrogradations qui vont à l'encontre même de leurs intérêts. En d'autres mots, tout projet de collaboration comporte le risque de masquer et d'affaiblir les intérêts conflictuels des travailleurs et d'ainsi les récupérer. Jusqu'à quel point peuvent-ils travailler de l'intérieur tout en étant matériellement, et donc structurellement, défavorables à de tels programmes ? Comment peuvent-ils à la fois participer à des stratégies de collaboration avec le patronat et soutirer le maximum pour les travailleurs, sans pour autant abandonner leurs intérêts contraires et conflictuels ? C'est là le défi à relever.

\section{Références bibliographiques}

ADAM, B.D., The Survival of Domination : Inferiorization and Everyday Life, New York, Elsevier, 1978.

Armstrong, P. et H. Armstrong, The Double Chetto: Canadian Women and Their Segregated Work, Toronto, McClelland and Stewart, 1978. 
Braverman, H., Labour and Monopoly Capital: The Degradation of Work in the Twentieth Century, New York, Monthly Review Press, 1974.

BrovermaN, I.K. et al., "Sex role stereotypes and clinical judgments and mental health ", Journal of Consulting and Clinical Psychology, vol. 34 no 1, 1970.

Chodorow, N., The Reproduction of Mothering, Berkeley, University of California Press, 1978.

Corrigan, P. et P. LeOnARD, "Production and reproduction", dans : Social Work Practice under Capitalism : A Marxist Approach, London, MacMillan, 1978 : 63-77.

Desmarais, D., "Stress, santé mentale et syndicalisme», Revue internationale d'action communautaire, 7/47, 1982: 179-186.

Gall, S., "The housewife", dans : R. Fraser (éd.), Work, London, Penguin, 1968 : 150-166.

GalPER, J.H., Social Work Practice : A Radical Perspective, chapitres 6 et 7, "An Orientation to Radical Practice" et "Direct Service», Englewood Cliffs (N.J.), Prentice Hall, 1980 : 109-155.

LEONARD, P., "Towards a paradigm for radical practice", dans: R. BAILEY et M. BRAKE (éds), Radical Social Work, London, Edward Arnold, 1975 : 46-61.

LEONARD, P., "Personality development within capitalism: Entering the social order ", Personality and Ideology: Towards a Materialist Understanding of the Individual, London, MacMillan, 1984 : 121-150.

Levine, H. et A. Estable, The Power Politics of Motherhood : A Feminist Critique of Theory and Practice, Ottawa, Centre for Social Welfare Studies, Université Carleton, 1981. ("Occasional Paper ».)

MeIsner, M. et al., "No exit for wives: Sexual division of labour and the culmination of household demands ", The Canadian Review of Sociology and Anthropology, vol. 12, no 4, 1975 : 424-439.

Moreau, M.J., "A structural approach to social work practice", The Canadian Journal of Social Work Education/Revue canadienne de service social, vol. 5, no 1, 1979 : 78-94.

MOREAU, M., "L'approche structurelle familiale en service social : le résultat d'un itinéraire critique", Revue internationale d'action communautaire, $7 / 47$, 1982: 159-172.

Nunes, M. et D. White, The Lace Ghetto, Toronto, New Press, 1972.

OAKLEY, A., The Sociology of Housework, New York, Pantheon, 1974.

RYAN, W., "Diswashers trained here : Ideology and education", dans : Equality, New York, Vintage Books, 1982: 121-137.

Shatz, E., The Defense of Socially Structured Inequality: A Theoretical Analysis, thèse de doctorat non publiée, Ann Arbor (Michigan), Université Brandeis, The Florence Heller Graduate School for Advanced Studies in Social Welfare, University Microfilms, 1973.

SRINIVAS, K.M., "Humanization of worklife in Canada : Progress, perspectives and prospects ", Journal of Occupational Behaviour, vol. 1, no 2, 1980, p. 107.

SteINER, C., Scripts People Live ; Transactional analysis of life scripts, chapitres 11, 17, 21, et 22: "Rescue: The Banal Scripting of Powerlessness", "Power Plays", "Strategies of Script Analysis", "The Therapy of the Three Basic 
Scripts : The Therapy of Depression " qui traitent des scripts de "Mindlessness, Lovelessness et Feelinglessness ", New York, Grove Press, 1974: 146-153; $212-222 ; 251-278$. 